\title{
Ideal Convergence in Generalized Topological Molecular Lattices
}

\author{
Kamal El-Saady ${ }^{1}$, Fatima Al-Nabbat ${ }^{2}$ \\ ${ }^{1}$ Department of Mathematics, Faculty of Science at Qena, South Valley University, Qena, Egypt \\ ${ }^{2}$ Department of Mathematics, College of Science, King Faisal University, Al-Hasa, Saudi Arabia \\ Email: el-saady@lycos.com, fatima math20@yahoo.com
}

Received 12 July 2015; accepted 4 September 2015; published 7 September 2015

Copyright (C) 2015 by authors and Scientific Research Publishing Inc.

This work is licensed under the Creative Commons Attribution International License (CC BY).

http://creativecommons.org/licenses/by/4.0/

(c) (i) Open Access

\begin{abstract}
The convergence theory of ideals in generalized topological molecular lattices is studied. Some properties of this kind of convergence are investigated. Finally, the relations between convergence theories of both molecular nets and ideals in GTMLs are discussed together with the $G_{2}$ separation axiom.
\end{abstract}

\section{Keywords}

Generalized Topological Molecular Lattice, Continuous GOH, Convergence of Molecular Nets, Convergence of Ideals

\section{Introduction}

After Wang [1] introduced the theory of topological molecular lattices or TMLs for short, several authors established various kinds of convergence theory in TMLs by using a corresponding concept of remote neighborhoods (see e.g. [2], [3]-[5]). The theory of remote neighborhood has been established first by Wang [1] as a dual notion of Pu and Liu's theory of the quasi-coincident neighborhoods in fuzzy topology [6] [7].

In [8], we introduced a generalization of Wang's topological molecular lattice called generalized topological molecular lattice or briefly GTML and studied the convergence theory of molecular nets by using the concept of generalized remote neighborhoods in these spaces.

In this paper, we aim to study the convergence of ideals in GTMLs and investigate the relations among this notion and that of molecular nets. Moreover, we state the relations with other defined topological notions in GTMLs such as generalized order homomorphism or GOH for short.

The paper is organized as follows. In Section 2, we will review some useful concepts in the paper. In Section 3, we will study the convergence in GTMLs in terms of ideals and investigate some properties of such conver- 
gence. Furthermore, we show the relations between convergence of ideals and the continuity of GOHs. In Section 4, we will discuss the relations between convergence of molecular nets and convergence of ideals in TMLs. Finally, Section 5 presents our conclusions.

\section{Preliminaries}

This section is devoted to recall some useful concepts which is required in the sequel. Let $L$ be a complete lattice with the smallest element $\perp$ and the largest element $\top$, an element $a \in L$ is said to be a molecule (some time called co-prime or join-irreducible) if for $b, c \in L, a \leq b \vee c$ then $a \leq b$ or $a \leq c$. The set of all molecules in $L$ is denoted by $M(L)$. The subset $B \subseteq L$ is called a minimal family of $a$ [1], if the following two conditions are hold:

a) $a=\vee B$.

b) If $D \subseteq L$ and $a \leq \vee D$, then $\forall b \in B, \exists d \in D$ such that $d \geq b$.

The greatest minimal family of $a$ is denoted by $\beta(a)$ while $\beta^{*}(a)=\beta(a) \cap M(L)$.

Throughout this paper, the entry $L(M)$ denotes a molecular lattice, that is a lattice $L$ and the set of its molecules $M$. For a non empty subset $I$ of a complete lattice $L, I$ is said to be an ideal [9], if it satisfies the following conditions:

a) For $a \in I, x \in L$ and $x \leq a \Rightarrow x \in I$.

b) For all $a, b \in I \Rightarrow a \vee b \in I$.

c) $T \notin I$.

Definition 2.1 [8] Let $L(M)$ be a molecular lattice. A subfamily $\eta \subset L$ is said to be a generalized closed topology, or briefly, generalized co-topology, if

$\left(T_{1}\right) \quad \eta$ is closed under arbitrary intersections;

$\left(T_{2}\right) \quad T \in \eta$.

The pair $(L(M), \eta)$ is called a generalized topological molecular lattice, or briefly, GTML.

Definition 2.2 [8] Let $(L(M), \eta)$ be a GTML, $a \in M, F \in \eta$, and $a \not \leq F$. Then $F$ is said to be a generalized remote neighborhood of $a$. The set of all generalized remote neighborhoods of a will be denoted by $\eta(a)$.

Recall that according to the definition of ideals, the family $\eta(a)$ is not necessary be an ideal in GTMLs while the family $\eta^{*}(a)=\{F \in \eta(a): \forall H \in \eta(a), F \vee H \in \eta(a)\} \subset \eta(a)$ satisfies the ideal conditions.

For a GTML $(L(M), \eta)$ and $A \in L$, the intersection of all $\eta$-elements containing $A$ is called the generalized closure of $A$ and denoted by $A^{-}$. that is,

$$
A^{-}=\wedge\{F \in \eta: A \leq F\}
$$

Definition 2.3 [8] Let $(L(M), \eta)$ be a GTML, $a \in M, A \in L$, then $a$ is said to be an adherence point of $A$, if for all $F \in \eta(a)$, we have $A \not F$.

It is clear that $a$ is an adherence point of $A$ if and only if $a \leq A^{-}$.

Definition 2.4 [1] Let $L_{1}$ and $L_{2}$ be complete lattices. A mapping $f: L_{1} \rightarrow L_{2}$ is said to be a generalized order homomorphism or GOH for short if

a) $f(a)=\perp$ if and only if $a=\perp$.

b) $f$ is join preserving, i.e; $f\left(\vee_{j} a_{j}\right)=\vee_{j} f\left(a_{j}\right)$.

c) $f^{\vdash}$ is join preserving, where $\forall b \in L_{2}, \quad f^{\vdash}(b)=\left\{a \in L_{1}: f(a) \leq b\right\}$.

Definition 2.5 [8] Let $\left(L_{1}\left(M_{1}\right), \eta_{1}\right)$ and $\left(L_{2}\left(M_{2}\right), \eta_{2}\right)$ be GTMLs and $f: L_{1} \rightarrow L_{2}$ be a GOH, then $f$ is called:

1) continuous GOH, if for every $H \in \eta_{2}$, we have $f^{\vdash}(H) \in \eta_{1}$.

2) continuous at a molecule $a \in M_{1}$, if for every $H \in \eta_{2}(f(a))$, we have $\left(f^{\vdash}(H)\right)^{-} \in \eta_{1}(a)$.

For a directed set $D$ and $A \in L$, the mapping $S: D \rightarrow M$ is called a molecular net and denoted by $S=\{S(n): n \in D\}$. The molecular net $S$ is said to be in $A$, if $S(n) \leq A, \forall n \in D$.

The molecular net $S$ is said to be:

1) eventually in $A$ if there exists $n_{0} \in D$ such that $\forall n \in D, n \geq n_{0}$, we have $S(n) \leq A$.

2) frequently in $A$ if for all $n \in D$ there exists $n_{0} \in D$ such that $n \geq n_{0}, S\left(n_{0}\right) \leq A$.

Definition 2.6 [8] Let $(L(M), \eta)$ be a GTML, $S=\{S(n): n \in D\}$ be a molecular net and $a \in M$, then:

1) $a$ is called a limit point of $S$, if $\forall F \in \eta(a), S(n)\lfloor F$ eventually true, and denoted by $S \rightarrow a$. The join 
of all limit points of $S$ will be denoted by $\lim S$.

i.e, $\lim S=\vee\{x \in M: S \rightarrow x\}$.

2) $a$ is called a cluster point of $S$, if $\forall F \in \eta(a), S(n) \not \subset F$ frequently true, and denoted by $S \propto a$. The join of all cluster points of $S$ will be denoted by cluS .

i.e, $\operatorname{cluS}=\vee\{x \in M: S \infty 0 x\}$.

Definition 2.7 [8] Let $(L(M), \eta)$ be a GTML, then $(L(M), \eta)$ is said to be $a G T_{2}$, if $\forall a, b \in M, a \wedge b=\perp$, there exists $H \in \eta^{*}(a)$ and $F \in \eta^{*}(b)$ such that $H \vee F=T$.

\section{Convergence of Ideals in GTMLs}

The aim of this section is to study the convergence in GTMLs in terms of ideals and investigate some properties of such convergence. Furthermore, we show the relations between convergence of ideals and the continuity of GOHs.

For the sake of convenience and no confusion, throughout this section and forwards, we restrict the attention of generalized remote neighborhoods of an element $a$ in GTMLs into the set $\eta^{*}(a)$ instead of $\eta(a)$.

Definition 3.1 Let $(L(M), \eta)$ be a GTML, $I \subset L$ be an ideal of $L$ and $a \in M$, then

1) $a$ is said to be a limit point of $I$ if $\eta^{*}(a) \subseteq I$, denoted by $I \rightarrow a$. In this case, we say that $I$ converges to a.

The join of all limit points of $I$ will be denoted by $\operatorname{limI}$.

2) $a$ is said to be a cluster point of $I$ if $\forall F \in \eta^{*}(a)$ and $\forall A \in I$, we have $F \vee A \neq \top$, denoted by $I \infty a$. In this case, we say that $I$ accumulates to $a$.

The join of all cluster points of $I$ will be denoted by cluI .

As a consequence, we obtain the following proposition:

Proposition 1 Let $(L(M), \eta)$ be a GTML, $I$ and $J$ be ideals of $L$ with $I \subset J$ and $a, b \in M$. Then we have:

1) $I \rightarrow a \Rightarrow J \rightarrow a$.

2) $J \propto a \Rightarrow I \infty a$.

3) $I \rightarrow a, b \leq a \Rightarrow I \rightarrow b$.

4) $I \infty a, b \leq a \Rightarrow I \infty b$.

Proof.

1) Let $I \rightarrow a$, then $\forall F \in \eta^{*}(a), F \in I \subset J$. Thus, $F \in J$ and hence $\eta^{*}(a) \subseteq J$.

Therefore, we have $J \rightarrow a$.

2) Let $J \propto a$, then $\forall F \in \eta^{*}(a)$ and $\forall A \in J$, we have $F \vee A \neq \top$.

Since $I \subset J$, then $B \in I \Rightarrow B \in J$ and hence $\forall F \in \eta^{*}(a)$ and $\forall B \in I, \quad F \vee B \neq \top$.

Therefore, we have $I \propto a$.

3) Let $I \rightarrow a$, then $\eta^{*}(a) \subseteq I$. Since $b \leq a$, then we get $\eta^{*}(b) \subseteq \eta^{*}(a)$. So, $\eta^{*}(b) \subseteq I$.

Therefore, we have $I \rightarrow b$.

4) Let $I \infty a$, then $\forall F \in \eta^{*}(a)$ and $\forall A \in I, F \vee A \neq \top$. But $\eta^{*}(b) \subseteq \eta^{*}(a)$, then $\forall H \in \eta^{*}(b)$ and $\forall A \in I$, we have $H \vee A \neq \top$. Therefore, we have $I \infty b$.

Theorem 2 Let $(L(M), \eta)$ be a GTML, I be an ideal of $L$ and $a \in M$, then

1) $I \rightarrow a$ if and only if $a \leq \lim I$.

2) $I \infty a$ if and only if $a \leq$ cluI.

Proof.

1) Let $I \rightarrow a$, by the definition of $\lim I=\vee\{x \in M: I \rightarrow x\}$, it is clear that $a \leq \lim I$.

Conversely, let $a \leq \lim I$ and $F \in \eta^{*}(a)$, then $a \leq F$ and hence $\lim I \leq F$. So, there exists $b \in M$ such that $I \rightarrow b, b \leq F$, then $F \in \eta^{*}(b)$. Thus, we have $\eta^{*}(a) \subseteq \eta^{*}(b)$ but $\eta^{*}(b) \subseteq I$, hence $\eta^{*}(a) \subseteq I$. Therefore, $I \rightarrow a$.

2) Let $I \infty a$, then similarly to 1$), a \leq$ cluI is clear.

Now, let $a \leq$ cluI and $F \in \eta^{*}(a)$, than $a \not F$ and hence cluI $₫ F$. So, there exists $b \in M$ such that $I \infty b, b \leq F$, then $F \in \eta^{*}(b)$. Thus, for all $A \in I, F \vee A \neq \top$, also, we have $\eta^{*}(a) \subseteq \eta^{*}(b)$. So, $\forall F \in \eta^{*}(a), \forall A \in I, F \vee A \neq \top$. Therefore, $I \infty a$.

Corollary 1 Let $(L(M), \eta)$ be a GTML, I be an ideal of $L$ and $a \in M$, then

1) $I \rightarrow a$ if and only if $\forall b \in \beta^{*}(a), I \rightarrow b$. 
2) $I \infty a$ if and only if $\forall b \in \beta^{*}(a), I \infty b$.

Theorem 3 Let $(L(M), \eta)$ be a GTML, $A \in L$, and $a \in M$, then $a \leq A^{-}$if and only if there exists an ideal $I$ in $L$ such that $A \notin I$ and $I \rightarrow a$.

Proof. $(\Rightarrow)$ Since $a \leq A^{-}$, then $a$ is an adherence point of $A$, i.e; $\forall F \in \eta^{*}(a), A \leq F$.

Put $I_{\eta^{*}(a)}=\left\{B \in L: \exists F \in \eta^{*}(a), B \leq F\right\}$, then $I_{\eta^{*}(a)}$ is an ideal and clearly that $A \notin I_{\eta^{*}(a)}$ also, we have $\eta^{*}(a) \subseteq I_{\eta^{*}(a)}$ which implies $I_{\eta^{*}(a)} \rightarrow a$.

$(\Leftarrow)$ Let $I \rightarrow a$, then $\forall F \in \eta^{*}(a)$, we have $\eta(a)^{*} \subseteq I$, i.e; $F \in I$. Since $A \notin I$, then $A \not F$. So, by Definition 2.3, $a$ is an adherence point of $A$ and hence $a \leq A^{-}$.

Lemma 1 Let $\left(L_{1}\left(M_{1}\right), \eta_{1}\right)$ and $\left(L_{2}\left(M_{2}\right), \eta_{2}\right)$ be GTMLs, $f: L_{1} \rightarrow L_{2}$ be a GOH, and I be an ideal in $L_{1}$. Then the set

$$
f(I)=\left\{B \in L_{2}: \exists A \in I, \text { s.t, } \forall a \in M_{1}, a \pm A \Rightarrow f(a) \npreceq B\right\}
$$

is an ideal in $L_{2}$.

Proof. It is easily to check the conditions of ideals.

Theorem 4 Let $\left(L_{1}\left(M_{1}\right), \eta_{1}\right)$ and $\left(L_{2}\left(M_{2}\right), \eta_{2}\right)$ be GTMLs, $f: L_{1} \rightarrow L_{2}$ be a continuous $G O H$ at $a \in M_{1}$ and I be an ideal in $L_{1}$. If $I \rightarrow a$, then $f(I) \rightarrow f(a)$.

Proof. Let $f$ be a continuous GOH at $a \in M_{1}$ and $I$ be an ideal in $L_{1}$ with $I \rightarrow a$, then $\forall H \in \eta_{2}^{*}(f(a))$, we have $\left(f^{\vdash}(H)\right)^{-} \in \eta_{1}^{*}(a) \subseteq I$. Hence, we get that $f^{\vdash}(H) \in I$ and for every $a \leq f^{\vdash}(H) \Rightarrow f(a) \leq H$. so, $H \in f(I)$ which implies that $\eta_{2}^{*}(f(a)) \subseteq f(I)$.

Therefore, $f(I) \rightarrow f(a)$.

Theorem 5 Let $\left(L_{1}\left(M_{1}\right), \eta_{1}\right)$ and $\left(L_{2}\left(M_{2}\right), \eta_{2}\right)$ be GTMLs, $f: L_{1} \rightarrow L_{2}$ be a GOH, then $f$ is continuous $G O H$ if and only if for every ideal $I$ of $L_{1}, f(\operatorname{limI}) \leq \operatorname{limf}(I)$.

Proof. $(\Rightarrow)$ Let $I$ be an ideal of $L_{1}, a \in M_{1}$ such that $I \rightarrow a$, hence $a \leq \operatorname{limI}$. We need to show that $f(a) \leq \lim f(I)$. Since $f$ is a continuous GOH, then $f$ is continuous at $a \in M_{1}$ and $f(a) \in M_{2}$. Hence, by Theorem 4, we get $f(I) \rightarrow f(a)$, i.e; $f(a) \leq \operatorname{limf}(I)$.

Since $f$ is a GOH, then $f$ preserves arbitrary joins and hence $f(\operatorname{limI}) \leq \operatorname{limf}(I)$.

$(\Leftarrow)$ We want to prove that $f$ is continuous at every $a \in M_{1}$, i.e; $\forall F \in \eta_{2}^{*}(f(a))$, we have $\left(f^{\vdash}(F)\right)^{-} \in \eta_{1}^{*}(a)$.

Assume that $a \leq\left(f^{\vdash}(F)\right)^{-}$. Hence, there exists an ideal $I$ such that $I \rightarrow a$ and $f^{\vdash}(F) \notin I$. Then $a \leq \lim I$ which implies that $f(a) \leq f(\lim I) \leq \operatorname{limf}(I)$. Thus, $f(I) \rightarrow f(a)$.

So, $\forall F \in \eta_{2}^{*}(f(a))$, we have $F \in f(I)$. By the definition of $f(I)$, there exists $A \in I$ such that $\forall a \in M_{1}, a\left\lfloor A \Rightarrow f(a) \nless F\right.$ equivalently that $a \not A A \Rightarrow a \leq f^{\vdash}(F)$. Hence, $f^{\vdash}(F) \leq A$ but $A \in I$, so $f^{\vdash}(F) \in I$. Contradiction.

Then, $a \not\left(f^{\vdash}(F)\right)^{-}$and hence $\left(f^{\vdash}(F)\right)^{-} \in \eta_{1}^{*}(a)$.

Therefore, $f$ is continuous GOH.

\section{Relations between Convergence of Molecular Nets and Convergence of Ideals in GTMLs}

In [3] and [5], the authors introduced a comparison between convergence of molecular nets and convergence of ideals in TMLs. In similar way, we discuss the relations between them in GTMs.

For a generalized topological molecular lattice $(L(M), \eta)$, let $I$ be an ideal in $L$, then the set

$$
D(I)=\{(a, A): a \in M, A \in I, a \leq A\}
$$

is a directed set with respect to the relation " $\leq$ "defined as

$$
\forall(a, A),(b, B) \in D(I),(a, A) \leq(b, B) \Leftrightarrow A \leq B
$$

Set $S(a, A)=a$, then the set 


$$
S(I)=\{S(a, A):(a, A) \in D(I)\}
$$

is a molecular net in $L(M)$ called the molecular net generated by the ideal $I$.

Now, let $S=\{S(n): n \in D\}$ be a molecular net in $L$, then the set

$$
I(S)=\{A \in L: S(n) \not A \text { eventually }\}
$$

is an ideal in $L$ called the ideal generated by $S$.

Theorem 6 Let $(L(M), \eta)$ be a GTML, $x \in M, I$ be an ideal in $L$ and $S$ be a molecular net in $L$, then we have

1) $S(I) \rightarrow x \Leftrightarrow I \rightarrow x$, (resp. $S(I) \infty x \Leftrightarrow I \infty x)$.

2) $I(S) \rightarrow x \Leftrightarrow S \rightarrow x$.

3) $I=I(S(I))$.

Proof. 1) Case I: Let $S(I) \rightarrow x$, then $\forall F \in \eta^{*}(x), S(I) \nless F$ eventually, i.e; there exists $(a, A) \in D(I)$ such that $\forall(b, B) \in D(I),(b, B) \geq(a, A)$, we have $S(I)(b, B)=b \not F$. Hence, $\forall b \leq A$ we get $b \leq F$, so $F \leq A$ but $A \in I$ which implies that $F \in I$.

Therefore, $\eta^{*}(x) \subseteq I$ and $I \rightarrow x$.

Conversely, let $I \rightarrow x$, then $\forall F \in \eta^{*}(x), F \in I$. Since $x \leq F$, then $(x, F) \in D(I)$ and $\forall(a, A) \in D(I)$ such that $(a, A) \geq(x, F)$, we have $S(I)(a, A)=a \pm A$, but $A \geq F$, hence $S(I)(a, A)=a \pm F$. Thus, $S(I) \rightarrow x$.

Case II: Let $S(I) \infty x, F \in \eta^{*}(x)$ and $A \in I$, then there exists $a \in M$ with $a \leq A$. Thus, $(a, A) \in D(I)$, since $S(I) \infty X$, there exists $(b, B) \in D(I)$ such that $(b, B) \geq(a, A)$ and $S(I)(b, B)=b \pm F$. Since

$b \in M, b \leq B$, then $b \leq F \vee B$ but $A \leq B$, so $b \leq F \vee A$.

Thus, $\forall F \in \eta^{*}(x)$ and $A \in I, F \vee A \neq \top$. Therefore, $I \infty x$.

Conversely, we need to show that $\forall F \in \eta^{*}(x), S(I) \not F$ eventually. Let $I \propto x$, then $\forall F \in \eta^{*}(x)$ and $A \in I, F \vee A \neq \mathrm{T}$.

Now, $\forall(a, A) \in D(I)$, we have $F \vee A \neq \top$, therefore, $\exists b \in M$ such that $b \leq F$ and $b \leq A$. So, $(b, A) \in D(I)$ and $(b, A) \geq(a, A), S(I)(b, A)=b \pm F$.

Therefore, $S(I) \notin F$ frequently and $S(I) \infty x$.

2) Let $I(S) \rightarrow x$, then $\forall F \in \eta^{*}(x), F \in I(S)$. By the definition of $I(S)$, we have $S \leq F$ eventually which means that $S \rightarrow x$.

Conversely, let $S \rightarrow x$, then $\forall F \in \eta^{*}(x), S \leq F$ eventually. So, $F \in I(S)$, i.e, $\eta^{*}(x) \subseteq I(S)$ which means $I(S) \rightarrow x$.

3) Let $A \in I$, then there exists $a \in M$ such that $(a, A) \in D(I)$ and $\forall(b, B) \in D(I)$ with

$(b, B) \geq(a, A)$, we have $S(I)(b, B)=b \leq B$. But $B \geq A$, hence $S(I)(b, B)=b \leq A$, i.e; $S(I) \leq A$ eventually.

Thus, $A \in I(S(I))$ and $I \subseteq I(S(I))$.

Now, let $A \in I(S(I))$, then $S(I) \nless A$ eventually, i.e; there exists $(b, B) \in D(I)$ such that

$\forall(e, E) \in D(I),(e, E) \geq(b, B)$, we have $S(I)(e, E)=e \not A$. Since $e \not E$ and $E \geq B$, then $e \leq B \Rightarrow e \leq A$. Hence, $A \leq B$ and $B \in I$, then $A \in I$ and $I(S(I)) \subseteq I$.

Therefore, $I=I(S(I))$.

According to Theorem 6, one can get directly the following result:

Corollary 2 Let $(L(M), \eta)$ be a GTML, I be an ideal in $L$ and $S$ be a molecular net in $L$, then the following statements hold:

1) $\lim I=\lim S(I)$.

2) $\operatorname{cluI}=\operatorname{cluS}(I)$.

3) $\lim S=\lim I(S)$.

Theorem 7 Let $(L(M), \eta)$ be a GTML, I be an ideal in $L$ and $S$ be a molecular net in $L$, then we have $\operatorname{cluS} \leq \operatorname{cluI}(S)$.

Proof. Let $x \leq c l u S$, then $S \infty x$. So, we need to show that $S \infty x \Rightarrow I(S) \infty x$.

Now, $\forall F \in \eta^{*}(x), S \nless F$ frequently. Also, $\forall A \in I(S), S \nless A$ eventually and hence, $S \nless F \vee A$ frequently. So, $\forall F \in \eta^{*}(x)$ and $\forall A \in I(S)$, we get $F \vee A \neq \top$.

Therefore, $I(S) \infty x$ and hence, $x \leq \operatorname{cluI}(S)$.

In 1986, Yang [9] introduced the concepts of maximal ideals and universal nets. 
Definition 4.1 [9] An ideal I in a complete lattice $L$ is called a maximal ideal, if for each ideal $J$ in $L$ such that $I \subset J$, we have $I=J$.

Definition 4.2 [9] A molecular net $S$ in a complete lattice $L$ is called a universal net, if there exists a maximal ideal in $L$ such that $S$ is a subnet of $S(I)$.

Proposition 8 Let $(L(M), \eta)$ be a GTML and I be a maximal ideal in $L$, then

$$
\lim I=\text { cluI } .
$$

Proof. It is clear that $\operatorname{limI} \leq$ cluI . Now, we prove that $\operatorname{cluI} \leq \operatorname{limI}$.

Let $x \leq c l u I$, then $I \infty x$. Put $J=\left\{B \in L: \exists A \in I, F \in \eta^{*}(x), B \leq A \vee F\right\}$

Then $J$ is an ideal in $L$ and clearly that $I \subseteq J$ and $\eta^{*}(x) \subseteq J$.

Since $I$ is a maximal ideal in $L$, we get $I=J$, hence $\eta^{*}(x) \subseteq I$.

So, $I \rightarrow x$ and $x \leq \lim I$. Therefore, limI $=$ cluI .

Theorem 9 Let $(L(M), \eta)$ be a GTML, then the following conditions are equivalent:

(i) For every ideal $I, \exists x \in M$ such that $I \infty x$.

(ii) For every maximal ideal $I, \exists x \in M$ such that $I \rightarrow x$.

(iii) For every universal net $S, \exists x \in M$ such that $S \rightarrow x$.

Proof. (i) $\Rightarrow$ (ii) Let $I$ be a maximal ideal, by (i), $\exists x \in M$ such that $I \infty x$. Since, $I$ is a maximal, then by Proposition 8, we have $I \rightarrow x$.

(ii) $\Rightarrow$ (i) Let $I$ be an ideal, then there exists a maximal ideal $J$ with $I \subseteq J$ and $\exists x \in M$ such that $J \rightarrow x$. Hence, $\eta^{*}(x) \subseteq J$.

So, $\forall A \in I$ and $\forall F \in \eta^{*}(x), A \vee F \neq \top$. Thus, $I \infty x$.

(ii) $\Rightarrow$ (iii) Let $S$ be a universal net and $x \in M$, then by the definition, there exists a maximal ideal $I$ such that $S$ is a subnet of $S(I)$. By (ii), we have $I \rightarrow x$ and hence $S(I) \rightarrow x$. Therefore, $S \rightarrow x$.

(iii) $\Rightarrow$ (ii) Let $I$ be a maximal ideal, then $S(I)$ is a universal net, by (iii), $\exists x \in M$ such that $S(I) \rightarrow x$. Then, we get $I \rightarrow x$.

Lastly, we conclude this section by studying the relation between the ideal convergence and the $G T_{2}$ separation axiom in GTMLs.

Theorem 10 Let $(L(M), \eta)$ be a GTML, then it is $G T_{2}$, if and only if for every ideal I in $L$, limI contains no disjoint molecules.

Proof. $(\Rightarrow)$ Let $(L(M), \eta)$ be $G T_{2}, I$ be an ideal in $L$. Assume that $a, b \leq \operatorname{limI}$ with $a \wedge b=\perp$. Then there exists $H \in \eta^{*}(a)$ and $F \in \eta^{*}(b)$ such that $H \vee F=\mathrm{T}$. Since $I \rightarrow a$ and $I \rightarrow b$, we have that $\eta^{*}(a) \subseteq I$ and $\eta^{*}(b) \subseteq I$. Hence, $F, H \in I$ which implies that $T=H \vee F \in I$. Contradiction with the definition of $I$.

Therefore, limI contains no disjoint molecules.

$(\Leftarrow)$ Assume that $(L(M), \eta)$ is not $G T_{2}$, then $\exists a, b \in M$ with $a \wedge b=\perp$ and $\forall F \in \eta^{*}(a), H \in \eta^{*}(b)$, we have $F \vee H \neq \top$. Put

$$
I=\left\{A \in L: A \leq F \vee H, F \in \eta^{*}(a), H \in \eta^{*}(b)\right\}
$$

Then $I$ is an ideal in $L$ with $a \leq \lim I$ and $b \leq \operatorname{limI}$. Hence, $\operatorname{limI}$ contains two disjoint molecules $a, b \in M$ which contradicts the assumption. Therefore, $(L(M), \eta)$ is $G T_{2}$.

Corollary 3 Let $(L(M), \eta)$ be a GTML, then the following statements are equivalents:

a) $(L(M), \eta)$ is a $G T_{2}$.

b) For every molecular net $S$ and every $a, b \in \beta^{*}(\lim S)$, we have $a \wedge b \neq \perp$.

c) For every ideal $I$ in $L$ and every $a, b \in \beta^{*}(\operatorname{limI})$, we have $a \wedge b \neq \perp$.

\section{Conclusion}

In this paper, we introduced a convergence theory of ideals in generalized topological molecular lattices by using the concept of generalized remote neighborhoods and studied some of its characterization and properties. Then, we investigated the relations between the ideal convergence and the continuity of GOH in GTMLs. Finally, we discussed the relations among the convergence theories of both ideals and molecular nets and also the $G T_{2}$ separation axiom. 


\section{References}

[1] Wang, G. -J. (1992) Theory of Topological Molecular Lattices. Fuzzy Sets and Systems, 47, 351-376. http://dx.doi.org/10.1016/0165-0114(92)90301-J

[2] Chen, S.-L. and Wu, J.-R. (2000) SR-Convergence Theory in Fuzzy Lattices. Information Sciences, 125, $233-247$. http://dx.doi.org/10.1016/S0020-0255(99)00058-4

[3] Chen, Y. (1996) Convergence in Topological Molecular Lattices. Fuzzy Sets and Systems, 84, 97-102. http://dx.doi.org/10.1016/0165-0114(95)00224-3

[4] Bai, S.-Z. (1997) Q-Convergence of Nets and Weak Separation Axioms in Fuzzy Lattices. Fuzzy Sets and Systems, 88, 379-386. http://dx.doi.org/10.1016/S0165-0114(96)00085-1

[5] Bai, S.-Z. (1997) Q-Convergence of Ideals in Fuzzy Lattices and Its Applications. Fuzzy Sets and Systems, 92, 357363. http://dx.doi.org/10.1016/S0165-0114(96)00174-1

[6] Liu, Y.-M. and Luo, M.-K. (1997) Fuzzy Topology. World Scientific, Singapore City.

[7] Pu, P.-M. and Liu, Y.-M. (1980) Fuzzy Topology. I. Neighborhood Structure of a Fuzzy Point and Moore-Smith Convergence. Journal of Mathematical Analysis and Applications, 76, 571-599. http://dx.doi.org/10.1016/0022-247X(80)90048-7

[8] El-Saady, K. and Al-Nabbat, F. (2015) Generalized Topological Molecular Lattices. Advances in Pure Mathematics, 5, 552-559. http://dx.doi.org/10.4236/apm.2015.59051

[9] Yang, Z.-Q. (1986) Ideals in Topological Molecular Lattices. Acta Mathematica Sinica, 29, 276-279. 